of microscopic colitis (MC). Due to the association of PPIs with diarrhoea there is a risk of confounding bias due to increased investigation with colonoscopy and biopsies. This has resulted in a controversy in case-control studies with regard to using a control group from the background population (BP) or a control group with investigated chronic diarrhoea (ICD). This abstract evaluates the use of the null hypothesis for MC and PPIs with relation to published casecontrol studies of MC and PPIs and with discussion of potential mechanisms

Methods The Null Hypothesis for MC and PPIs can be categorised according to MC being largely clinical and investigated or a largely subclinical uninvestigated disease.

Hypothesis 1: PPIs and MC are unrelated and MC is always overt and investigated by colonic biopsies.

Hypothesis 2: PPIs and MC are unrelated and MC is always subclinical.

For age and sex matched groups - those with clinical MC (hypothesis 1) that are detected will have the same percentage on PPIs as those from the background population (BP), whereas those with subclinical MC (hypothesis 2) that are detected will have the same percentage on PPIs as those with investigated chronic diarrhoea (ICD)

Results There are 6 published case-control studies and a recent abstract that provide adjusted odds ratios (AORs)/odds ratios (ORs) for PPIs and MC. Some of the larger studies have divided MC patients into the two subgroups; collagenous colitis (CC) and lymphocytic colitis (LC).

\begin{tabular}{|c|c|c|c|c|c|c|c|}
\hline & & $\mathrm{n}$ & $\%$ & $\mathrm{BP} \%$ & $\mathrm{AOR} /$ & IDC\% & $\mathrm{AOR}$ \\
\hline & & & PPIs & PPIs & $\mathrm{OR}$ & PPIS & OR \\
\hline \multirow{3}{*}{$\begin{array}{l}\text { Bonderup } \\
2014\end{array}$} & $\mathrm{CC}$ & 3474 & 55.7 & 14.2 & 7.04 & & 3.47 \\
\hline & & & & & & & \\
\hline & LC & 2277 & 36.7 & 12.9 & 3.37 & & 3.57 \\
\hline Bonderup & $\mathrm{CC}$ & 6254 & & & 8.75 & & \\
\hline \multicolumn{8}{|l|}{2017} \\
\hline (abstract) & LC & 4398 & & & 5.03 & & \\
\hline \multirow[t]{2}{*}{ Banares 2013} & $\mathrm{CC}$ & 120 & 33.3 & 14.8 & & & \\
\hline & LC & 70 & 31.4 & 14.8 & & & \\
\hline Kesthelyi 2010 & $\mathrm{MC}$ & 95 & 37.9 & 12.6 & 4.5 & & \\
\hline Pascua 2010 & $\mathrm{MC}$ & 26 & 12 & 45 & 0.21 & 34 & 0.29 \\
\hline Verhaugh & MC & 1211 & 41.8 & 17.5 & 3.79 & & \\
\hline \multicolumn{8}{|l|}{2016} \\
\hline Masclee 2015 & $\mathrm{MC}$ & 218 & 30 & 2.4 & 7.3 & 6.3 & 10.6 \\
\hline
\end{tabular}

Conclusions MC is unlikely to be always investigated and there is some evidence that MC can be detected in asymptomatic individuals. However, it is also unlikely that $\mathrm{MC}$ is largely subclinical and not investigated. All but one case-control studies have shown increased AORs for MC and PPIs for BP controls and similarly in 2 of 3 studies for ICD controls. The only study not showing an association included only 26 cases with MC and a very high usage of PPIs in the control BP of $45 \%$. The mechanisms of how PPIs may cause MC are unclear but theories include increased intestinal epithelial permeability, alteration of colonic bacterial flora and increased production of collagen by colonocytes. The association of MC with medications including PPIs should not be ignored and cessation of potentially causative medications requires consideration.

\section{PWE-041 ALTERATION IN SMALL BOWEL MOTILITY, GUT PEPTIDES AND PATIENT'S SYMPTOMS IN ACTIVE CROHN'S DISEASE}

${ }^{1,2}$ Gordon Moran*, ${ }^{1,2}$ Asseel Khalaf, ${ }^{2,3}$ Caroline Hoad, ${ }^{4}$ Alex Menys, ${ }^{2}$ Shellie Radford, ${ }^{2}$ Adam Nowak, ${ }^{1,2}$ Stephen Paparo, ${ }^{2}$ Melanie Lingaya, ${ }^{2}$ Yirga Falcone, ${ }^{2}$ Gulzar Singh, ${ }^{4}$ Stuart Taylor, ${ }^{1,2}$ Robin Spiller, ${ }^{3}$ Penny Gowland, 1,2 Luca Marciani. 'Nottingham Digestive Diseases Centre, School of Medicine, University of Nottingham, Nottingham, UK; ${ }^{2}$ NIHR Biomedical Research Centre in Gastrointestinal and Liver Diseases at Nottingham University Hospitals NHS Trust and the University of Nottingham, Nottingham, UK; ${ }^{3}$ Sir Peter Mansfield Imaging Centre, School of Physics and Astronomy, University of Nottingham, Nottingham, UK; ${ }^{4}$ Centre for Medical Imaging, Division of Medicine, UCL, London, UK

\subsection{6/gutjnl-2018-BSGAbstracts. 173}

Introduction Intestinal inflammation in Crohn's disease (CD) is associated with an increase in Polypeptide YY (PYY), Glucagon-like peptide 1 (GLP-1) and cholecystokinin (CCK). CD patients experience postprandial fullness and nausea. These symptoms may be linked to the increase in plasma gut peptides levels and alterations in intestinal motility.

Our aims are to quantify gut peptide, small bowel motility and patient symptom response to a standard test meal using Magnetic Resonance Imaging (MRI).

Methods Subjects underwent baseline and postprandial MRI scans, symptom questionnaires and blood sampling (GLP-1, PYY, CCK) at intervals for $270 \mathrm{~min}$ following a test meal: soup (400 g) (Heinz, Wigan, UK); (kcal) 51, protein $1.5 \mathrm{~g}$, carbohydrate $4.7 \mathrm{~g}$, fat $2.9 \mathrm{~g}$ per $100 \mathrm{~g}$.

MRI scans were performed using a $1.5 \mathrm{~T}$ Philips Achieva MRI scanner. Gastric volume, small bowel water content (SBWC) and small bowel motility were assessed using MRI. Patients also underwent a standard contrast enhanced clinical MR enterography (MRE) and MaRIA score applied to quantify disease activity. All subjects gave informed written consent. Trial registration number: NCT03052465. Data is presented as mean \pm SEM.

Results CD patients showed a significantly $(\mathrm{p} \leq 0.05)$ slower

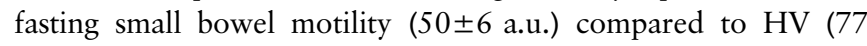
\pm 10 a.u.). Postprandial SBWC was significantly greater in CD than $\mathrm{HV}$ (measured as area under the curve CD: 18452, HV: 13760, p $\leq 0.05$ ). Fasting PYY (CD: $236 \pm 16$ pg/mL, HV: 118 $\pm 11 \mathrm{pg} / \mathrm{mL}, \mathrm{p} \leq 0.0001)$ and GLP-1 (CD: $50 \pm 8 \mu \mathrm{g} / \mathrm{mL}$ HV: 13 $\pm 3 \mu \mathrm{g} / \mathrm{mL}, \mathrm{p} \leq 0.0001)$ were significantly higher in CD compared to $\mathrm{HV}$ with this difference persisting at each time point of the study $(p \leq 0.0001)$. The meal induced a significant increase $(p \leq 0.0001)$ in fullness, bloating and abdominal pain scores in patients $(28 \pm 4 \mathrm{~mm}, 22 \pm 3 \mathrm{~mm}$ and $12 \pm 2 \mathrm{~mm}$ respectively) compared to $\mathrm{HV}(12 \pm 4 \mathrm{~mm}, 3 \pm 3 \mathrm{~mm}$ and 1 $\pm 2 \mathrm{~mm}$ respectively). No differences were noted in gastric volumes, CCK concentration and postprandial motility.

Conclusion The decrease in fasting small bowel motility noted in $C D$ may be ascribed to the increased fasting GI peptides. Understanding the physiological changes in disease groups will allow us to identify the key biomarkers for pharmacological modulation to improve patient symptoms. 